\title{
Limitações acerca da implantação de softwares do Sistema Único de Saúde na Atenção Básica
}

\author{
Josélia Batista Dias de Souza \\ Administradora pelo Centro Universitário de Goiás. Docente substituta na área de administração pela \\ Universidade Estadual de Goiás (UEG). Servidora Pública Municipal. Especialista em Finanças pela UNESA; em \\ Gestão Pública pela UFG e em Gestão Pública Municipal pela UnB. Mestre em Gestão e Auditoria Ambiental- \\ Engenharia e Tecnologia Ambiental pela Unini Puerto Rico. Discente Especial no Programa de Mestrado \\ Profissional em Gestão Organizacional da Universidade Federal de Catalão (PPGGO/UFCat) \\ $\square$ joseliabd@gmail.com \\ Thiago Fernandes Borges \\ Administrador. Assistente da Engenharia de Programas e bill of material (BOM de Produção) da HPE \\ Automotores do Brasil. Discente Especial no Programa de Mestrado Profissional em Gestão Organizacional da \\ Universidade Federal de Catalão (PPGGO/UFCat) \\ $\square$ thiagoborges2112@gmail.com

\section{Marcos Fernandes-Sobrinho} \\ Físico pela Universidade Federal de Uberlândia (UFU) e Administrador pela Faculdade de Administração de \\ Brasília (FAAB). Doutor em Educação em Ciências e Matemática pela Universidade Brasília (UnB). Docente \\ permanente da área de Física e no PPGEnEB do Instituto Federal Goiano (IF Goiano) e no PPGGO da \\ Universidade Federal de Catalão (UFCat) \\ $\bowtie \underline{\text { marcos.sbf@gmail.com }}$
}

Recebido em 17 de setembro de 2020

Aceito em 23 de março de 2020

\section{Resumo:}

O presente artigo busca analisar as principais limitações inerentes à implantação dos softwares CDS e PEC no âmbito da gestão da informação em saúde. No intuito de se chegar a esses propósitos que seguem uma abordagem qualitativa aplicou-se diferentes métodos. Inicialmente, empregou-se no campo da tipologia e dos procedimentos metodológicos: a pesquisa bibliográfica e a pesquisa documental em bases relacionadas ao tema, e em segundo momento, aplicou-se a pesquisa de campo a partir da realização de entrevista semiestruturada com profissionais que atuam no setor de Atenção Básica de um município de pequeno porte, localizado ao norte do Estado de Goiás. As reflexões em literaturas pertinentes conduzem ao entendimento da essencialidade desses softwares para a melhoria do processo de coleta, de atualização e de aproveitamento de dados dos cidadãos a fim de se promover um atendimento integralizado aos mesmos na esfera da saúde pública primária. Enfim, temse a compreensão de que tais sistemas apresentam-se como inovações incrementais para o processo informacional do SUS. Por intermédio da investigação compreendeu-se que os sistemas estão sendo implantados de forma gradual nas Unidades Básicas de Saúde do local, e apesar disso, tem-se limitações relacionadas ao acesso de recursos tecnológicos e à capacitação profissional, de forma que entraves como esses colaboram para que estes avanços ainda permaneçam incipientes em territórios demograficamente menores no Brasil.

Palavras-chave: Tecnologia da Informação, Sistema Único de Saúde, e-sus aB, Softwares CDS e PEC. 


\title{
Limitations on the implementation of software from the Unified Health System in Primary Care
}

\begin{abstract}
:
This article seeks to analyze the main limitations inherent to the implementation of CDS and PEC software in the scope of health information management. In order to achieve these purposes that follow a qualitative approach, different methods were applied. Initially, in the field of typology, methodological procedures, bibliographic research and documentary research were used in bases related to the theme, and secondly, field research was applied based on a semi-structured interview with professionals working in the field. Primary Care sector of a small municipality, located in the north of the State of Goiás. The reflections in pertinent literature lead to an understanding of the essentiality of these softwares for the improvement of the process of collecting, updating and making use of citizens' data in order to promote comprehensive care to them in the sphere of primary public health. Finally, there is an understanding that such systems are presented as incremental innovations for the SUS information process. Through the investigation, it was understood that the systems are being implemented gradually in the Basic Health Units of the place, and despite this, there are limitations related to access to technological resources and professional training, so that obstacles such as these collaborate so that these advances still remain incipient in demographically smaller territories in Brazil.
\end{abstract}

Keywords: Information Technology, Health Unic System, e-SUS AB, CDS and PEC software.

\section{Limitaciones en la implementación de software del Sistema Único de Salud en Atención Primaria}

\section{Resumen:}

Este artículo busca analizar las principales limitaciones inherentes a la implementación del software CDS y PEC en el ámbito de la gestión de la información sanitaria. Para lograr estos propósitos que siguen un enfoque cualitativo, se aplicaron diferentes métodos. Inicialmente, los procedimientos metodológicos, la investigación bibliográfica y la investigación documental se utilizaron en el campo de la tipología sobre bases relacionadas con el tema, y en segundo lugar, la investigación de campo se aplicó en base a una entrevista semiestructurada con profesionales que trabajan en el campo. Sector de Atención Primaria de un pequeño municipio, ubicado en el norte del estado de Goiás. Las reflexiones en la literatura pertinente conducen a la comprensión de la esencialidad de estos softwares para mejorar el proceso de recopilación, actualización y uso de los datos de los ciudadanos con el fin de promover una atención integral para ellos en el ámbito de la salud pública primaria. Finalmente, se entiende que dichos sistemas se presentan como innovaciones incrementales para el proceso de información del SUS. A través de la investigación, se entendió que los sistemas se están implementando gradualmente en las Unidades Básicas de Salud del lugar, y a pesar de esto, existen limitaciones relacionadas con el acceso a los recursos tecnológicos y la capacitación profesional, por lo que obstáculos como estos colaboran para que estos avances sigan siendo incipientes en territorios demográficamente más pequeños en Brasil.

Palabras clave: Tecnología de la Información, Sistema único de Salud, e-SUS AB, Softwares CDS y PEC.

\section{INTRODUÇÃo}

Vive-se em um mundo cada vez mais integrado e exigente quanto ao uso de novas Tecnologias da Informação e Comunicação (TIC's) nos diversos contextos que envolvem relações sociais, econômicas e políticas. 
É importante destacar que as TIC's são compreendidas como soluções constituídas por recursos computacionais, capazes de produzir, de armazenar, de transmitir, e de garantir a segurança e o acesso às informações para diferentes públicos da sociedade (SILVA; SOARES, 2018). Em consonância com isso, observa-se que as TIC's terminam por estabelecer uma nova forma de se relacionar com o mundo, sendo essas um imperativo para a alteração da dinâmica nas relações já frisadas (CAMPANÁRIO, 2002).

Essa necessidade de adaptação acompanha a vida de todos nós, está especialmente presente na gestão de diferentes cenários organizacionais que encontram nestes recursos possibilidades de melhorar as relações de consumo, a qualidade dos bens e serviços ofertados no mercado. Enfim, esse movimento tecnológico de ordem globalizada agrega propósitos dos seguimentos compreendidos na esfera privada e também no contexto público, principalmente no que reporta-se às atividades desenvolvidas no âmbito da gestão em saúde, uma vez que, as TIC's, nesse contexto tornaram-se cada vez mais importantes para um atendimento mais amplo e facilitado ao cidadão (SANTOS; FERREIRA, 2015).

Assim, consideramos a importância de se estudar sobre a Coleta de Dados Simplificada (CDS) e o Prontuário Eletrônico do Cidadão (PEC) os quais são softwares de tecnologia da informação que fazem parte do Sistema Único de Saúde (SUS). Necessariamente, são empregados dentro do chamado e-SUS Atenção Básica (e-SUS AB), e juntos se mostram como metodologias dinâmicas de coleta de dados, que além de registrarem as informações dos cidadãos, dos domicílios, e dos atendimentos realizados pelos profissionais da $\mathrm{AB}$, ainda podem ser úteis para o planejamento e entre outras medidas na gestão da saúde pública.

O e-SUS AB é entendido como um software que compõe o Sistema de Informação em Saúde (SIS), sendo empregado como uma estratégia capaz de quantificar e qualificar informações no âmbito da Atenção Básica, sendo também, um instrumento útil para agilizar o trabalho de profissionais de saúde e de gestores desta área (ALVES et al, 2017).

Por conseguinte, o sistema tecnológico dos softwares do e-SUS AB aparece como medida para gerar maior informatização dos dados da população e para contribuir com a integralização dos atendimentos ao público. Portanto, temos uma questão a ser respondida ao longo desse estudo: Qual tem sido a dinâmica e as limitações para a implantação dessas tecnologias da informação preconizadas pelo SUS em um município brasileiro de pequeno porte? 
A partir de estudo realizado em Alves et al (2017), chega-se a percepção de que as unidades federativas municipais nem sempre possuem estruturas e condições adequadas para a efetivação de políticas tecnológicas, ainda que essas medidas colaborarem para o desenvolvimento social e para a qualidade de vida populacional.

Vale ressaltar que as políticas tecnológicas, por sua vez, são identificadas como medidas que emergem-se no contexto da gestão pública, em especial, para garantir que o uso das tecnologias torne-se um processo realmente possível de acontecer, criando-se condições de segurança, armazenamento e acesso de qualidade às informações pelos usuários dos sistemas disponíveis (LASTRES; CASSIOLATO, 2003).

O desenvolvimento desse estudo foi incitado pelo interesse referente à evolução da tecnologia da informação no âmbito do SUS, especialmente no que toca ao sistema e-SUS AB e aos seus softwares CDS e PEC que se apresentam como os mais recentes métodos para o registro sistemático e dinâmico de dados dos cidadãos (BRASIL, 2016 a).

Por outro lado, entendeu-se ser fundamental a busca pelo conhecimento dos impactos desse processo na prática dos trabalhadores do SUS nos municípios, bem como o entendimento relacionado a maneira pela qual esses recursos tecnológicos estão sendo introduzidos e aproveitados para o gerenciamento de dados e aprimoramento do serviço em saúde.

Assim, o objetivo geral deste estudo é analisar as principais limitações inerentes à implantação dos softwares do sistema e-SUS AB no âmbito da gestão da informação em saúde. Em Consonância, temos como objetivos específicos: evidenciar os principais conceitos e teorias relacionadas aos softwares do e-SUS e suas contribuições para a melhoria do sistema de informação em saúde; identificar a realidade vivenciada pela gestão e profissionais da atenção primária à saúde que atuam realmente com essas tecnologias no município alvo, estabelecendo ao final um diálogo reflexivo frente aos principais achados.

Para tanto, na perspectiva da pesquisa qualitativa, trabalhamos especialmente com as pesquisas bibliográfica e documental, por meio de informações sobre o assunto em artigos e trabalhos anteriores, bem como na legislação vigente e documentos oficiais que abordam sobre o assunto. Também aplicamos a pesquisa de campo com levantamentos junto a 
profissionais que atuam com os programas num município de pequeno porte no Estado de Goiás.

De certo modo, o estudo evoca alguns dos limites que fazem parte das realidades de diversas unidades de atendimento básico no Brasil quanto ao contato com as novas tecnologias da informação, as quais se fazem necessárias, no entanto, ainda não se tem plenas condições para a implementação de medidas criadas no âmbito governamental brasileiro com o intuito de melhorar o processo informativo em saúde.

\section{REFERENCIAL TEÓRICO}

\section{Aspectos do uso da tecnologia da informação na era do conhecimento pelo Sus}

Diversos estudos realizados nas últimas décadas apontam cada vez mais a essencialidade da tecnologia da informação para o desenvolvimento econômico, social, político e cultural da sociedade, especialmente a partir do fenômeno da globalização, de modo que, o setor de saúde não desvincula-se deste processo. (CAMPENÁRIO, 2002).

De tal maneira, as TIC's, que formam um conjunto de recursos computacionais direcionados para a promoção da informação, no âmbito da saúde, são fundamentais à geração de um processo de trabalho qualitativo e eficiente (DIAS, 2016).

Em estudos de Lemos (2000), percebeu-se que a adesão da inovação para as transformações do sistema de comunicação e de informação é um efeito que ocorre de maneira acelerada por parte dos agentes econômicos que desejam manter a sua competitividade no mercado.

O interesse pelas tecnologias da informação por parte do SUS, por exemplo, foi iniciado desde a sua implantação nos anos 80, uma vez que, desde então, evidenciava-se a necessidade de melhorar a comunicação com o usuário, vislumbrando-se formas mais eficazes de coleta e tratamento de dados, bem como uma melhor oferta de saúde à população brasileira (BRASIL, 2016 a).

Apesar desses ideais, por muito tempo o trabalho do SUS foi desenvolvido a partir do emprego de um número considerável de recursos manuais, enfim, de formulários padrões 
utilizados em hospitais, em Unidades Básicas de Saúde (UBS), inclusive no que diz respeito às vinculadas à Estratégia da Saúde da Família (ESF), e noutros ambientes de atendimento para baixa, média e alta complexidade (COSTA; ACAMPORA, 2017).

Contudo, na Atenção Primária à Saúde, desde a sua criação, a demanda por procedimentos diferenciados potencializou um maior uso de sistemas de informação, até mesmo porque os atendimentos nesta área possuem maior abrangência às necessidades dos cidadãos, e consequentemente fazem interação e acompanhamento contínuo das famílias em determinado espaço territorial (BRASIL, 2015).

Em todo caso, o SUS que é uma política de Estado fundamentada na promoção do atendimento integral ao paciente tem se atentado para a urgência de um sistema de informação cada vez mais flexível às demandas dos usuários, levando-se em conta as suas individualidades e a complexidade que envolve a pessoa humana (BRASIL, 2017).

A preocupação em obter o uso das tecnologias para comunicar-se e para aproximarse das mais diversas parcelas da sociedade brasileira, tendo em vista as dificuldades geográficas e o crescimento populacional, erigiu na esfera do SUS diversas medidas para tornar o atendimento ao público mais eletrônico possível (DANTAS; CURIOSO, 2016).

Com isso, tivemos a reestruturação do processo de trabalho desse sistema público, em que se percebe a criação e implantação da informatização de dados através dos seguintes programas que compreendem o e-Saúde, a saber, o Cartão Nacional de Saúde (CNS), o e-SUS Hospitalar, o e-SUS Atenção Básica, o e-SUS SAMU, o Barramento da Saúde, o e-SUS Especialidades, e o Registro Eletrônico em Saúde Nacional (BRASIL, 2015).

No entanto, uma vez que neste estudo o nosso foco são os softwares constituídos pelas últimas alterações em torno do sistema de informação no contexto da saúde primária, é importante entendermos a instauração desse processo. Assim, tem-se que através da Portaria no 1.412, aprovada em 10 de julho de 2013 (BRASIL, 2013) houve a instituição do Sistema de Informação em Saúde para a Atenção Básica (SISAB), a partir do qual estabeleceu-se que:

Art. $2^{\circ}$ A operacionalização do SISAB será feita por meio da estratégia do Departamento de Atenção Básica (DAB/SAS/MS) denominada e-SUS Atenção Básica (e-SUS AB).

$\S 1^{\circ}$ A estratégia e-SUS AB é composta por dois sistemas de "software" que instrumentalizam a coleta dos dados que serão inseridos no SISAB: 
Portanto, desde que instituído o SISAB evidenciou-se a criação e a implementação pelo setor de informática do Departamento de Atenção Básica (DAB) dos softwares CDS e PEC, que são objetos de estudo nesta reflexão, relacionada à inovação na área de sistemas de informação em saúde.

É notável considerar que no trabalho com informações na Estratégia da Saúde da Família (ESF), tornou-se evidente a implementação no final dos anos 90 do chamado Sistema de Informação de Atenção Básica (SIAB) (BRASIL, 1998) que precedeu o atual SISAB.

O SIAB, por sua vez, ainda é frequente em várias unidades de atendimento primário no Brasil, mas aos poucos vem dando espaço ao seu sucessor que se coloca mais dinâmico e aplicável às necessidades de informatização dos trabalhos em saúde integralizada (COSTA; ACAMPORA, 2017).

Enquanto o SISAB possibilita a economicidade no uso de papel e de processos manuais (DIAS, 2016), o SIAB baseou-se por muito tempo no trabalho com fichas impressas conhecidas como: "Ficha A, Ficha B-GES, Ficha B-HA, Ficha B-DIA, Ficha B-TB, Ficha B-HAN, Ficha C, Ficha D" e com registros de coleta e consolidação de dados denominados de "Relatórios [...] SSA2, SSA4, PMA2, PMA4, A1, A2, A3, A4", pelos quais se registravam informações referentes aos públicos de Atenção Primária (BRASIL, 1998, p. 4).

O SUS, de uma forma geral, vem preconizando a inovação tecnológica em seu contexto de atuação junto à população brasileira, inclusive a partir do respeito a diretrizes da Política Nacional de Informação e Informática em Saúde (PNIIS) que ajudam a nortear ações relacionadas à Tecnologia da Informação e Comunicação (TIC), isso no intuito de se ter maior condição de enfrentar os problemas de saúde e atender ao usuário (BRASIL, 2016 a).

Em suma, tem-se que as políticas de informação do SUS não surgiram unicamente da vontade de se ter um sistema para evidenciar-se a necessidade do usuário, mas também este advento sofreu influências de propostas internacionais (BRASIL, 2015). Nesse sentido, a globalização e a necessidade de se seguir os ditames do sistema de tecnologia mundial são elementos cruciais que impulsionaram ainda mais à aderência de transformações na estrutura informacional dos serviços nacionais em saúde. 


\section{O sistema tecnológico do e-SUS AB: características inerentes aos softwares CDS e PEC}

Como já destacado, o e-SUS AB ganhou forma a partir da instituição do SISAB, em que acredita-se por intermédios de fundamentos elucidados em Oliveira e Raminelli (2014), que a própria Lei de Acesso à Informação (LAI - nº 12.527/2011) potencializou ainda mais os interesses em se ter sistemas informacionais mais transparentes e capazes de possibilitar maior integração entre governo e cidadãos.

No entanto, como destacam Santos e Ferreira (2012), não se pode tirar o mérito que há nas políticas de informação do SUS de realmente promoverem a melhoria dos processos de gestão de dados em saúde, bem como garantir ao profissional e ao usuário facilidades e flexibilidades durante os atendimentos públicos.

Pelo e-SUS AB, a gestão em saúde brasileira acabou por incrementar um processo inovador (CAMPANÁRIO, 2002), no qual se transforma um sistema de informação, repleto de plataformas e terminologias, numa plataforma única de dados, de maneira que as suas estratégias consistem em: individualizar o registro do cidadão, reduzir os processos de retrabalho na coleta de dados junto ao paciente, informatizar unidades de saúde, e coordenar o processo de cuidado (BRASIL, 2015).

Assim, voltemos para dar enfoque ao CDS e ao PEC enquanto principais instrumentos de coleta de informação no cenário atual da Atenção Básica dentro da perspectiva da inovação tecnológica dos sistemas de saúde pública.

Ambos os softwares de acordo com as definições do SISAB devem ser oferecidos gratuitamente, pela pactuação entre os entes federativos, obedecendo-se a um cronograma de envio de informações pelas equipes de $A B$ às bases do sistema, e ainda, o processo de implantação no Distrito Federal e nos municípios deve levar em conta as particularidades e cenários locais, sendo um processo dinâmico e gradual (BRASIL, 2013).

A princípio, é importante considerar que o CDS permite o registro, num mesmo ambiente tecnológico, do trabalho de agentes comunitários de saúde, de odontólogos, de enfermeiros, médicos, e técnicos em enfermagem, bem como da equipe do Núcleo de Atenção à Saúde da Família (NASF), entre outros, de sorte que os dados podem ser lançados de modo 
off-line, sendo os dados posteriormente enviados via exportação de arquivos ao PEC (DIAS, 2016).

O PEC, por sua vez, permite o registro dos mesmos dados do CDS, porém de forma digital (online), de maneira que este possibilita o Registro Clínico Orientado por Problemas (RCOP) e a adesão à Classificação Internacional de Atenção Primária (CIAP), carecendo-se de que o software esteja instalado numa UBS, a qual deve ser habilitada como ambiente central de trabalho, uma vez que, ao final, os dados coletados periodicamente são transmitidos diretamente para a base do SISAB através de tal recurso (DUTRA et al, 2017).

Com isso, analisa-se que os softwares do e-SUS funcionam de forma on-line e off-line, de maneira a descentralizarem o acesso em diferentes localidades (UBS, secretarias de saúde, residências, etc.) pelos profissionais vinculados à AB (BRASIL, 2017).

\section{Contribuições e desafios desse sistema}

Temos que esses softwares são meios de coleta, de registro e de gestão de dados e informações vinculadas ao cidadão no âmbito da saúde pública, logo, estes são importantes instrumentos que possibilitam o acesso às políticas de Atenção Primária a partir de um processo inovador pelo uso da tecnologia na oferta do serviço (OLIVEIRA et al, 2016).

O e-SUS $A B$ a partir dessas ferramentas tecnológicas alia-se ao processo de reestruturação dos sistemas de informação do Ministério de Saúde (MS) permitindo a qualificação da gestão informacional, a ampliação da qualidade nos atendimentos integrais aos cidadãos, bem como possibilitam a informatização no intuito de se promover um SUS eletrônico, mais dinâmico e acessível (SANTOS; FERREIRA, 2015).

No entanto, a partir de compreensão de Alves et al (2017) tem-se que o CDS e o PEC são softwares vinculados ao desenvolvimento tecnológico da informação no contexto da saúde que ainda encontram-se em gradual implantação nas equipes de saúde da família, apesar de que já é uma exigência de que todos os municípios brasileiros adéquem ao uso dos mesmos.

De certo modo, nota-se a carência de qualificação profissional, de recursos tecnológicos de informação e entre outros para que os propósitos do atendimento 
individualizado e personalizado ao cidadão torne-se realmente possível nos atendimentos da Atenção Primária à saúde no Brasil (DIAS, 2016).

\section{METODOLOGIA}

Com base no que é apontado por Creswel (2007), é possível valer-se de diferentes métodos especialmente no que se reporta a pesquisas de cunho qualitativo para chegar-se aos resultados pretendidos, caracterizando-se isso pela aplicação de uma metodologia mista.

Assim, o presente estudo valeu-se dessa diversidade de métodos para atingir os propósitos da pesquisa, no período de setembro a novembro de 2018. De modo que, seguindo também orientações metodológicas das quatro tipologias da pesquisa científica apontadas por Silveira e Córdova (2009) utilizou-se: quanto à abordagem: a pesquisa qualitativa; quanto à natureza: a pesquisa aplicada; quanto aos objetivos: a pesquisa descritiva; quanto aos procedimentos: a pesquisa bibliográfica, a pesquisa documental e a pesquisa de campo, de maneira que, essas coexistem de forma interdependente nesta investigação.

Inicialmente fez-se a busca em bancos de dados como SciELO e Google acadêmico/Google Scholar, buscando-se fundamentos teóricos em artigos e em documentos oficiais eletrônicos e impressos, estando isso vinculado ao emprego da pesquisa bibliográfica e da pesquisa documental. Sendo que, por meio dessas buscou-se evidenciar os principais conceitos e teorias relacionadas ao processo de adesão do SUS às inovações tecnológicas tangentes aos sistemas de informação em saúde, especialmente aos softwares do sistema e-SUS AB e suas contribuições para a melhoria da gestão da informação nesta área.

Em segundo momento, empregou-se a pesquisa de campo no intuito de identificar os limites da realidade vivenciada pelos gestores e profissionais quanto à implantação dessas tecnologias do e-SUS no município alvo (GIL, 2002). Tal processo foi desenvolvido pela aplicação de um roteiro de entrevista semiestruturado (SILVEIRA; CÓRDOA, 2009), o qual foi composto por duas partes: a primeira contendo três questões inerentes à caracterização dos indivíduos (idade, sexo, profissão) e a segunda tendo quatro questões específicas em relação ao assunto (conforme apêndices A e B). 
A entrevista terminou por abranger o seguinte público, constituído por quatro participantes: uma integrante vinculada à equipe da coordenação da Atenção Básica da localidade (gestão), uma digitadora, a qual tem como função basilar lançar no sistema e-SUS $\mathrm{AB}$ os dados referentes as produções de outros profissionais atuantes na Estratégia Saúde da Família e que possuem limitações tecnológicas; uma recepcionista, que agenda consultas nesta mesma plataforma, e um técnico do SUS, responsável pela implantação do sistema eSUS na localidade, tendo esses entrevistados a faixa etária compreendida entre 24 e 40 anos de idade.

Considera-se que dois desses participantes estão lotados na Unidade de Saúde modelo presente na zona urbana, e dois estão na coordenação de AB. Contudo, todos esses indivíduos realizam registros nos softwares (PEC ou CDS) das atividades desempenhadas por outros profissionais que estão na área urbana ou rural do município, tendo em vista a precariedade tecnológica ainda existente. Assim sendo, o principal critério para escolha dos participantes foi a relação desses com o trabalho potencializado pelos softwares em análise, levando-se, ainda, em consideração a disponibilidade de cada sujeito em participar do estudo. Nisso, utilizou-se o Termo de Consentimento Livre e Esclarecido (TCLE), resguardando-se a imagem e os dados pessoais dos participantes, entre outros aspectos que demandam o sigilo e a ética da pesquisa.

Construiu-se ao final um diálogo reflexivo frente aos principais achados por meio da interação teórico-prática de modo a levantar evidências e possíveis sugestões para garantir a melhoria dessa inovação tecnológica no campo informativo da saúde municipal.

Os dados coletados foram analisados a partir do processo de análise de conteúdo, na modalidade qualitativa, fazendo-se as considerações a partir dos teóricos estudados ao longo do estudo, tais como: Almeida et al (2016), Alves et al (2017), Campanário (2002), Dantas e Curioso (2016), Dutra et al (2017), Oliveira et al (2016), entre outros.

Em relação ao ambiente dessa pesquisa, observa-se que o município localiza-se ao norte do Estado de Goiás, tendo uma vasta área de proteção ambiental, e ainda, possui elementos históricos e culturais que reforçam a sua peculiaridade frente às políticas públicas existentes. O lugar tem pouco mais de 9.000 habitantes, distribuídos entre a zona rural e a urbana, abrangendo a diferentes grupos étnicos culturais e raciais. 
Com isso, a localidade é constituída por vários elementos que lhe trazem a necessidade de aderir ao e-SUS AB, quando consideradas as definições de Costa e Acampora (2017). Assim, entre esses condicionantes, obtém-se que, com exceção de um hospital municipal, o município é atendido por: quatro UBS (sendo duas rurais e duas urbanas, uma das rurais tem seus atendimentos realizados na área urbana) que promovem a saúde da família a partir do Programa Mais Médicos, tendo-se, também, atendimentos no âmbito do Programa Saúde na Escola, e ainda, há uma academia de saúde, onde ocorre a atuação da equipe do Núcleo de Atenção à Saúde da Família. Enfim, ter o funcionamento desses estabelecimentos praticamente exige que o município adéque os softwares CDS e PEC em seu contexto de coleta e gestão das informações em saúde.

\section{RESULTADOS E DISCUSSÃo}

\section{Limites para a implantação dos softwares PEC e CDS do sistema do e-SUS AB}

Em princípio buscou-se investigar se os softwares do e-SUS AB, a saber, o CDS e o PEC já tinham sido implantados no município em estudo, sendo evidenciada a sua trajetória até o presente a partir de dados obtidos junto à representante da coordenação (gestão) de $\mathrm{AB}$ (Quadro1).

A trajetória de implantação dos sistemas no referido município em muito se assemelha ao que acontece em várias cidades brasileiras no que toca ao atendimento às diretrizes do SISAB que ocorre de forma gradual (BRASIL, 2013). Contudo, evidencia-se que na localidade o estágio deste processo pode ser classificado como implantado uma vez que aproximadamente 60\% dos ambientes passíveis de instalação dos softwares já estão atendidos (BRASIL, 2016 b). Destaca-se que, principalmente numa das unidades rurais do município em estudo, cujo funcionamento ocorre nas áreas de difícil acesso geográfico e tecnológico esse processo tecnológico torna-se ainda mais difícil de ser implementado. Ao contrário disso, a outra unidade, também rural, mas que faz o seu atendimento aos pacientes no perímetro urbano já obteve essa tecnologia, ainda que também subutilizada pelos profissionais de AB. 
Quadro 1 - Trajetória da implantação dos softwares no município (2014 a 2018)

\begin{tabular}{|c|c|c|}
\hline ANO & CDS & PEC \\
\hline 2014 & $\begin{array}{l}\text { O CDS começou a ser implantado somente para o } \\
\text { lançamento da produção dos funcionários, ficando } \\
\text { o mesmo restrito ao contexto da secretaria } \\
\text { municipal de saúde da localidade, sendo utilizado } \\
\text { por dois digitadores. }\end{array}$ & Ainda não implantado. \\
\hline 2017 & $\begin{array}{l}\text { O CDS passou a ser empregado em uma das quatro } \\
\text { Unidades Básicas de Saúde da ESF do município. }\end{array}$ & Ainda não implantado. \\
\hline \multirow[t]{2}{*}{2018} & $\begin{array}{l}\text { O CDS foi multiplicado para o uso na recepção de } \\
\text { duas unidades e noutros ambientes, especialmente } \\
\text { para facilitar o processo de lançamento de } \\
\text { cadastros individuais e domiciliares de moradores, } \\
\text { partindo-se de informações repassadas pelos ACS. }\end{array}$ & $\begin{array}{l}\text { No mês de agosto, foi implantado } \\
\text { o PEC em três unidades } \\
\text { (representadas no meio urbano), } \\
\text { sendo o trabalho com essa } \\
\text { tecnologia mais facilitado do que } \\
\text { inicialmente. }\end{array}$ \\
\hline & \multicolumn{2}{|c|}{$\begin{array}{l}\text { Atualmente há } 09 \text { profissionais que já fazem o uso de ambos os softwares ou de pelo } \\
\text { menos um deles no processo de informação em saúde na localidade. }\end{array}$} \\
\hline
\end{tabular}

Fonte: Elaborado pelos autores com base nos dados obtidos nas entrevistas.

Em tangência à forma pela qual vem acontecendo o uso desses softwares no município em estudo identificou-se por meio dos entrevistados que:

a) O uso ocorre gradualmente desde 2014 do CDS;

b) Nem todas as unidades estão atendidas com o processo tecnológico, mas a maioria já está relacionando-se com essa nova tecnologia da informação. Ressalta-se que mesmo tendo essa implantação dos sistemas na maior parte das unidades, o quantitativo de profissionais que as utilizam é mínimo.

c) Atualmente pelos programas já são realizados os seguintes registros: cadastro domiciliar (imóveis habitados ou não), cadastro individual dos moradores/pacientes usuários do SUS, visitas domiciliares dos ACS, atendimentos odontológicos, atendimentos médicos, atendimentos de enfermeiros e agendamento de consulta;

d) Os funcionários das UBS/ESF estão em processo de adaptação e de aceitação dessa nova tecnologia, tendo-se resistências, bem como, dificuldades em torno do 
conhecimento tecnológico por profissionais de diferentes níveis de escolaridade, ocupação profissional e local de domicílio (rural ou urbano);

Essas características fazem menção ao que já observamos quanto à implantação dessas tecnologias no município em estudo, o que termina por associar-se ao que Dias (2016) qualificou como limites para a promoção do SUS eletrônico no Brasil. E ainda, tem-se que apesar de carências tecnológicas e da falta de capacitação dos servidores tanto para a aceitação quanto para a adequação a esta nova estrutura de trabalho, as unidades estão conseguindo sobressair especialmente no que tange à tentativa de acompanhar as inovações (CAMPANÁRIO, 2002), mesmo tendo-se a submissão a um processo que acontece de forma similar ao que se chama de demanda empurrada, ou seja, a adesão a esta tecnologia acaba por ser compulsiva (LASTRES; CASSIOLATO, 2003).

Contudo, em entrevista com a coordenação de $A B$, com um técnico do programa (esses dois primeiros atuam de forma geral no atendimento de todas as Unidades Básicas do município quanto às demandas relacionadas aos softwares), com uma digitadora e uma recepcionista, ambas atuantes na UBS modelo, e atendem respectivamente no lançamento de dados de produções de outros profissionais e nos agendamentos de consultas de pacientes da zona urbana e rural, evidenciou-se que tais softwares possuem as seguintes limitações e desafios no município:

a) OCDS, que tem uma maior facilidade de distribuição e implantação é um programa lento e que demonstra erros em sua configuração, carecendo de ajustes e atualizações constantes;

b) O PEC, que possui funcionalidades mais avançadas e consegue ter uma velocidade maior, bem como maior eficiência no trabalho de registro de dados termina por ser de difícil distribuição, uma vez que esse somente pode ser instalado nas UBS ou na secretaria municipal de saúde;

c) O CDS é utilizado de forma off-line por qualquer funcionário credenciado, já o PEC depende de se estar on-line (conectado à rede de internet); 
d) A conexão precária à internet atrapalha no desenvolvimento dos trabalhos especialmente no PEC;

e) Há carência de capacitação dos usuários atuais e daqueles em potencial dos programas, por exemplo: médicos, odontólogos e técnicos em higiene bucal, enfermeiros, técnicos de enfermagem, recepcionistas, agentes comunitários de saúde, recepcionistas, coordenação de $\mathrm{AB}$, e secretário de saúde. Tal situação faz com que a relação com as tecnologias aconteça especialmente por técnicos e digitadores.

f) O desenvolvimento dos trabalhos nos softwares depende entre outros da qualidade dos dados coletados pelos ACS que em maioria não possuem recursos e nem orientações específicas para tal;

g) Por limitações da própria população, os registros acabam sendo incompletos pela falta do cartão nacional do SUS (CNS), de dados pessoais diversos como: número do PIS, CPF, RG, endereço, contato e entre outros;

h) A ausência de integração do sistema com outros órgãos e informações acaba por fazer com que os registros dos usuários sejam subutilizados;

i) As unidades rurais, principalmente a que fica em área de difícil acesso não está conseguindo acompanhar os processos pela carência no acesso à energia elétrica ou alternativa, que é um meio básico para adesão à tecnologia (DANTAS; CURIOSO, 2016).

Os entraves considerados pelos profissionais, logo acima, quanto ao uso dos softwares terminam por apontar o quanto na prática ainda há necessidades de adaptações às realidades locais durante a implantação e a utilização desses recursos nas unidades de atendimento em saúde primária (OLIVEIRA et al, 2016). As informações em tópicos, acima, evidenciam que apesar de se tratar de sistemas que vislumbram maior descentralização da informação para a melhoria do acesso do cidadão aos serviços básicos, na localidade, o CDS é percebido como a ferramenta mais acessível, porém com maiores dificuldades tendo em vista a constante necessidade de atualização (DUTRA et al, 2017). Por outro lado, o PEC, que a partir da versão 2.0 tem tido menores erros, é obrigatoriamente restrito ao trabalho interno nas unidades, sendo este centralizador das informações enviadas ao SISAB, conduzindo-as diretamente ao MS (COSTA; ACAMPORA, 2017). 
Outros elementos que colaboram para a restrição das atividades dentro dos softwares é a carência de capacitação profissional (DIAS, 2016), dando-se vazão às situações de estranhamento à inovação incremental no desenvolvimento da integralização das informações pelo SUS no local.

A partir das entrevistas com os participantes deste estudo obteve-se a compreensão desses quanto à importância dos softwares CDS e PEC para a melhoria do processo de gestão da informação em saúde na localidade, portanto, evidenciou-se que tais processos:

a) Contribuem para economia de papel e redução da desordem nas UBS/ESF;

b) Contribui para o maior aproveitamento do espaço nas unidades;

c) São fundamentais para que o município passe a arrecadar mais recursos para a saúde a partir de um número real de moradores;

d) Facilita e ajuda no acesso a um relatório mais completo da saúde do paciente;

e) Permite um maior controle dos gastos públicos em saúde, em princípio dos gastos com medicação (controle de estoque);

f) São processos dinâmicos que vem se adaptando a necessidade dos usuários;

g) Estão cada vez mais atualizados e na medida em que alcançar a excelência demorarão mais para requisitar novas versões ou backup (BRASIL, 2017);

h) Tendem a ser cada vez mais multiplicados no intuito de permitir que o usuário consiga gerenciar seus próprios atendimentos, a depender da situação de cada um;

i) Otimizam o trabalho de gestão da informação em saúde de uma maneira ampla no contexto de $\mathrm{AB}$.

A partir dos profissionais, tem-se que esses reconhecem a relevância do CDS e do PEC para a melhoria do processo de gestão da informação, sendo observado por eles alguns elementos, que na verdade, fazem parte da estratégia do e-SUS AB, erigindo-se a possibilidade de que através de tais processos tecnológicos diminua-se o retrabalho, informatize-se as unidades de saúde, individualize-se o registro e o atendimento à população, ao mesmo tempo em que promova-se e coordene-se o cuidado ao paciente (BRASIL, 2016). 
Enfim, esses softwares já estão sendo entendidos como ferramentas imprescindíveis para a melhoria da gestão da informação no SUS, confirmando-se o que já vem sendo defendido em estudos teóricos e práticos anteriores sobre este assunto.

\section{CONSIDERAÇÕES FINAIS}

Através do estudo, evidenciou-se a importância dos softwares CDs e PEC no processo de gestão informacional no âmbito do Sistema de Informação em Saúde para a Atenção Básica, sendo possível analisar os ideais teóricos da estratégia do e-SUS e também identificar os principais limites inerentes à implantação dessas tecnologias no âmbito municipal, como preconizado nos objetivos da pesquisa.

Em resposta à questão norteadora desse estudo (Qual tem sido a dinâmica e os limites da implantação dessas tecnologias da informação preconizadas pelo SUS em um município brasileiro de pequeno porte?), obteve-se que, como dinâmica, o município investigado tem conseguido implantar gradativamente os softwares em suas unidades passíveis de instalação e utilização dos mesmos.

Nesse sentido, vale observar que principalmente uma das UBS localizadas em áreas rurais, por não ter recursos mínimos para a implantação de qualquer forma de tecnologia, está entre as excluídas desse processo tecnológico. Em decorrência dessa situação, os profissionais que nesta atuam acabam dependendo ainda mais do trabalho de outros operadores como: recepcionista e digitadora, sobretudo para a realização dos lançamentos de suas produções nos softwares estudados.

Destarte, entre os principais limites para a efetividade dos ideais preconizados na geração de um SUS eletrônico encontram-se: a carência tecnológica em decorrência do baixo investimento em equipamentos básicos e carência de energia elétrica ou alternativa no caso dos estabelecimentos rurais.

Evidencia-se, também, a necessidade de haver a conscientização dos profissionais de saúde sobre a importância do sistema e-SUS para a melhoria da qualidade e da eficiência em seus trabalhos, principalmente no que refere-se à coleta de informações e registros 
produtivos. Nisso, considerando que, a introdução desses softwares deu-se de maneira imposta, o que trouxe resistência às mudanças por parte de profissionais atuantes nos diferentes níveis da $\mathrm{AB}$ local.

Nota-se, ainda, nesse mesmo sentido, a urgência de se ofertar maior e melhor capacitação profissional, tendo em vista que nos moldes apresentados, nem todos os profissionais executores dos atendimentos na Atenção Primária sentem-se seguros para operarem com autonomia tais programas, com isso, delegam as atividades de operacionalização para outros servidores.

Logo, importa salientar que, na prática, os próprios executores dos serviços é que deveriam operar os softwares para lançarem suas produtividades, e não intermediários como tem acontecido nesse município. Destaca-se que, principalmente os agentes comunitários de saúde, que atuam nas áreas rurais, os quais em maioria possuem pouca escolarização vivenciam ainda mais as limitações de acessibilidade e de capacitação, estando entre os que mais demandam intermediações no uso do e-SUS.

Em contramão, há entre os agentes comunitários atuantes nas áreas urbanas, aqueles que possuem alta escolaridade, bem como maior domínio tecnológico, e que conseguem operar com facilidade tais tecnologias, principalmente o software CDS, sem que haja a necessidade de intermediações de outros profissionais. Assim, os ACS urbanos, principalmente os que possuem estrutura tecnológica própria operam esse programa em suas próprias casas, tendo em vista não terem uma sala de apoio em suas unidades. Esses lançam suas produções e dados, bem como exportam e enviam os tais para a gestão de AB efetuar o lançamento direto no PEC central.

Se pensarmos no caso desses profissionais observados, talvez poderíamos incorrer na percepção de que a escolaridade, a urbanidade e o domínio do conhecimento tecnológico sejam fatores que colaboram para que os softwares sejam facilmente implementados e utilizados por toda a equipe de AB. No entanto, as situações de médicos, enfermeiros, odontólogos, técnicos de enfermagem, entre outros, acabam por apontarem que nem sempre esses elementos interferem totalmente na operacionalização do e-SUS.

A exemplo disso há o caso de médicos e enfermeiros, os quais embora ocupem funções de nível superior em suas atribuições e moram na zona urbana, esses possuem limitações 
tecnológicas muitas vezes semelhantes às daqueles que ocupam funções de nível médio ou moram em áreas rurais, vindo a necessitarem de igual modo do trabalho de recepcionistas e digitadores.

Portanto, no atual cenário, pelos dilemas associados, o ato de delegar a outros servidores (intermediários) o lançamento dos dados e produções daqueles profissionais (executores) que estão com dificuldades para operacionalizar o sistema e-SUS acaba por sobrecarregar a esses primeiros. Com isso, ao invés de se tornar o serviço mais célere e eficiente, acaba-se por torná-lo enfraquecido e oneroso. Além de que, os demais profissionais da equipe de $A B$ terminam por não familiarizarem-se com essa tecnologia, a qual é tão importante para a qualidade das informações no SUS.

Assim, é preciso que na gestão da saúde pública, esses elementos sejam considerados para se construir estratégias de enfrentamentos das dificuldades profissionais no âmbito dessa tecnologia.

É fundamental que esses e demais aspectos associados, sejam postos como prioridades dos principais gestores do SUS, considerando que se resolvidos, darão maiores condições para que a instrumentalização e a aplicação dos softwares do e-SUS AB, fazendo-os chegarem ao alcance de mais servidores, aumentando-se a eficiência dos registros e atendimentos.

Assim, teve-se condições de entender que no município pesquisado, assim como ocorre em diversas localidades brasileiras, esses sistemas ainda não estão em pleno funcionamento, uma vez que o processo de implantação tem acontecido de forma gradual, evidenciando-se conforme as diretrizes do SISAB as limitações e particularidades que envolvem a realidade de cada lugar.

Portanto, obtivemos que o trabalho com esses sistemas é fundamental para a garantia dos objetivos do SUS com a política de Atenção Primária à saúde, na qual acredita-se que a partir desses softwares ter-se-á condições de garantir registros que permitem um atendimento individualizado e integralizado ao cidadão em várias partes do País, vindo isso a colaborar para o planejamento e gestão de políticas relacionadas aos públicos de atendimento nesta esfera. 
Recomenda-se que, no município, assim como no caso de outras localidades brasileiras de pequeno porte, os gestores busquem apoios das instâncias federativas que possuem maiores recursos para a intervenção nos itens identificados, bem como possam lançar mão de parcerias com organizações diversas, entre elas, as universidades, que trabalham em pesquisas inovadoras e que possam ajudar em articulações externas capazes de contribuir com o processo de enfrentamentos desses limites fundamentais que compreendem tal realidade.

Essa pesquisa dentro dos estudos multidisciplinares e interdisciplinares é útil para que diferentes áreas possam ter fundamentações teóricas e práticas aplicadas à reflexão em torno do uso da tecnologia da informação no âmbito da gestão das organizações públicas na busca pela eficiência nos processamentos de dados do cidadão, principalmente, no que tange à oferta de atendimentos mais personalizados em saúde e que sejam capazes de atender às necessidades dos indivíduos na sociedade.

Enfim, o estudo aqui apresentado limitou-se na análise mais sucinta pertinente aos dilemas que estão envolvidos no processo de implementação do uso de softwares do e-SUS AB num município do Estado de Goiás. Contudo, este deixa como principais lacunas e limitações que merecem estudos futuros e mais aprofundados as seguintes: o acesso das tecnologias do SUS nas áreas rurais, a influência ou não da escolaridade na operacionalização tecnológica, e ainda, o investimento tecnológico em Unidades Básicas de Saúde de municípios de pequeno porte.

\section{REFERÊNCIAS}

ALMEIDA, M. J. G. G.; FIGUEIREDO, B. B.; SALGADO, H. C.; TORTURELLA, I. M. []. Discussão Ética sobre o Prontuário Eletrônico do Paciente. Revista Brasileira de Educação Médica, v. 40, n. 3, p. 521-527, 2016. DOI: http://dx.doi.org/10.1590/1981-52712015v40n3e01372015.

ALVES, J. P. ; DINIZ, I. V. A.; FRANÇA, K. T. G; SILVA, L. M; MARTINIANO, C. S. Avanços e desafios na implantação do e-SUS-Atenção Básica. In: II Congresso Brasileiro de Ciências da Saúde, 2017, 10 p. Disponível em: http://www.editorarealize.com.br/revistas/conbracis/trabalhos/TRABALHO_EV071_MD4_SA7_ID788_150520 17202831.pdf. Acesso em: 07 set. 2018.

BRASIL. Ministério da Saúde. Informativo e-SUS AB. Coordenação Estadual da Atenção Básica-Estratégia Saúde da Família/DAS/SES-RS, 2017. Disponível em: https://atencaobasica.saude.rs.gov.br/upload/arquivos/201701/11144305-informativo-e-sus-ab.pdf. Acesso em: 20 ago. 2018. 
- Ministério da Saúde. Secretaria-Executiva. Departamento de Monitoramento e Avaliação do SUS. Política Nacional de Informação e Informática em Saúde. Ministério da Saúde, Secretaria-Executiva, Departamento de Monitoramento e Avaliação do SUS. Brasília: Ministério da Saúde, 2016 a, 56 p. Disponível em: http://bvsms.saude.gov.br/bvs/publicacoes/politica_nacional_infor_informatica_saude_2016.pdf. Acesso em: 07 set. 2018.

. Ministério da Saúde. Secretaria de Atenção à Saúde. E-SUS Atenção Básica: Manual do Sistema com Coleta de Dados Simplificada: CDS - Versão 2.1 [recurso eletrônico] / Ministério da Saúde, Secretaria de Atenção à Saúde, Secretaria-Executiva. - Brasília: Ministério da Saúde, 2016 b, 171 p.

. Ministério da Saúde. Secretaria de Atenção à Saúde. Departamento de Regulação, Avaliação e Controle. Sistemas de Informação da Atenção à Saúde: Contextos Históricos, Avanços e Perspectivas no SUS/Organização Pan-Americana da Saúde. Brasília, 2015, 166 p.

. Portaria no 1.412, de 10 de julho de 2013. Institui o Sistema de Informação em Saúde para a Atenção Básica (SISAB). Disponível em: http://www.cosemsrn.org.br/wp-content/uploads/2013/07/PORTARIA-No1.412-DE-10-DE-JULHO-DE-2013-Institui-o-Sistema-de-Informação-em-Saúde-para-a-Atenção-BásicaSISAB...2.pdf. Acesso em: 20 set. 2018.

SIAB: manual do sistema de informação de atenção básica / Secretaria de Assistência à Saúde, Coordenação de Saúde da Comunidade. Brasília: Ministério da Saúde, 1998. 98 p.

CAMPANÁRIO, M. A. Tecnologia, Inovação e Sociedade. In: Seminário VI Módulo da Cadeira CTS I Colômbia, chamado de Inovação Tecnológica, Economia e Sociedade. Organização dos Estados Ibero-Americanos para a Educação, Ciência e Cultura (OEI) / Instituto Colombiano para o Desenvolvimento da Ciência e Tecnologia da Colômbia (Colciencias), setembro de 2002. Disponível em: https://www.oei.es/historico/salactsi/milton.htm. Acesso em: 20 ago. 2018.

COSTA, C. A. V.; ACAMPORA, L. Como alimentar o SISAB através do e-SUS AB quando o município possui sistema próprio? Florianópolis: DAB/MS, 2017.

CRESWELL, J.W. Projeto de pesquisa: métodos qualitativo, quantitativo e misto. 2.ed. Porto Alegre: Artmed, 2007.

DANTAS, A.; CURIOSO, R. Geografia e saúde: o lugar como premissa da atividade informacional da Atenção Básica do Sistema Único de Saúde.

Saúde Soc. São Paulo, v.25, n.3, p.721-735, 2016. DOI 10.1590/S0104-12902016152646.

DIAS, D. Processo de implantação da estratégia e-SUS AB na região ampliada de saúde oeste de minas gerais. Universidade Federal de São João Del Rei, 2016.

DUTRA, D. M. P.; MOREIRA, T. M. M.; SOUSA FILHO, R. T.; DUARTE, D. O. P. Prontuário Eletrônico do Cidadão (PEC): a experiência de implantação como orientação aos gestores e técnicos do processo de implantação do eSUS/PEC- Atenção Básica, 2017.

GARBIN, H. B. R. ; GUILAM, M. C. R.; PEREIRA NETO, A. F. Internet na promoção da saúde: um instrumento para o desenvolvimento de habilidades pessoais e sociais. Physis Revista de Saúde Coletiva, Rio de Janeiro, 22 [1]: 347-363, 2012. DOI: https://doi.org/10.1590/S0103-73312012000100019.

GIL, A. C. Como elaborar projetos de pesquisa. 4. ed. - São Paulo: Atlas, 2002.

LASTRES, H. M. M.; CASSIOLATO, J. E. Glossário de Arranjos e Sistemas Produtivos e Inovativos Locais. Arranjos produtivos locais: uma nova estratégia de ação para o SEBRAE. Rede de Pesquisa em Sistemas Produtivos e Inovativos Locais, novembro, 2003. Disponível em: www.ie.ufrj.br/redesist. Acesso em: 17 ago 2018. 
LEMOS, C. A inovação na era do Conhecimento. In: Parcerias Estratégicas n. 8, maio 2000, p. 157-180. Disponível em: http://seer.cgee.org.br/index.php/parcerias_estrategicas/article/viewFile/104/97. Acesso em: 17 ago. 2018.

OLIVEIRA, A. E. C.; LIMA, I. M. B.; NASCIMENTO, J. A.; COELHO, H. F. C., SANTOS, S. R. Implantação do e-SUS AB no Distrito Sanitário IV de João Pessoa (PB): relato de Experiência. Saúde Debate | Rio de Janeiro, v. 40, n. 109, p. 212-218, Abr-Jun, 2016.

OLIVEIRA, R. S.; RAMINELLI, F. P. O Direito ao Acesso à Informação na Construção da Democracia Participativa: uma análise da página do Conselho Nacional de Justiça no Facebook. Sequência (Florianópolis), n. 69, p. 159-182, dez. 2014.

SANTOS, D. C.; FERREIRA, J. B. B. o prontuário da família na perspectiva da coordenação da atenção à saúde. Physis Revista de Saúde Coletiva, Rio de Janeiro, v.22, n. 3, p. 1121-1137, 2012.

SILVA, V. A.; SOARES, M. H. F. B. O uso das tecnologias de informação e comunicação no ensino de Química e os aspectos semióticos envolvidos na interpretação de informações acessadas via web. Ciênc. Educ., Bauru, v. 24, n. 3, p. 639-657, 2018. Disponível em: https://www.scielo.br/scielo.php?script=sci_abstract\&pid=S151673132018000300639\&lng=en\&nrm=iso\&tlng=pt. Acesso em: 15 mar. 2020.

SILVEIRA, D. T.; CÓRDOVA, F. P. Unidade 2 - a pesquisa científica. In.: GERHARDT, T. E.; SILVEIRA, D. T. Métodos de pesquisa. Coordenado pela Universidade Aberta do Brasil - UAB/UFRGS e pelo Curso de Graduação Tecnológica - Planejamento e Gestão para o Desenvolvimento Rural da SEAD/UFRGS. Porto Alegre: Editora da UFRGS, 2009, p. 31-42.

\section{(cc) Br}

Este trabalho está licenciado com uma Licença Creative Commons - Atribuição 4.0 Internacional. 